\title{
Efficient Robust Design Optimization of Optical Systems
}

\author{
Stephanie Kunath ${ }^{1, *}$ \\ ${ }^{1}$ Dynardo GmbH, Steubenstrasse 25, Weimar, 99423, Germany
}

\begin{abstract}
To accelerate the virtual product development of using optical simulation software, the Robust Design Optimization approach is very promising. Optical designs can be explored thoroughly by means of sensitivity analysis. This includes the identification of relevant input parameters and the modelling of inputs vs. outputs to understand their dependencies and interactions. Furthermore, the intelligent definition of objective functions for an efficient subsequent optimization is of high importance for multi-objective optimization tasks. To find the best trade-off between two or more merit functions, a Pareto optimization is the best choice. As a result, not only one design, but a front of best designs is obtained and the most appropriate design can be selected by the decision maker. Additionally, the best trade-off between output variation of the robustness (tolerance) and optimization targets can be found to secure the manufacturability of the optical design by several advanced approaches. The benefit of this Robust Design Optimization approach will be demonstrated.
\end{abstract}

\section{Motivation}

The optimization of advanced optical designs is very challenging due to their complexity, nonlinearity, a huge number of input parameters and interactions between them. At the same time, the demands for the performance of these systems are versatile and very high and even get higher, not only concerning optimization targets but also robustness criteria. Furthermore, totally new developments, like new materials, manufacturing possibilities and very short product development times, at the same time, require advanced methodologies to develop competitive optical products.

To accelerate the virtual product development using optical simulation software, additional Robust Design Optimization methods are available in Dynardos software optiSLang.

\section{Robust Design Optimization Methods}

\subsection{Sensitivity analysis}

Optical designs can be explored thoroughly by means of sensitivity analysis. This includes the identification of relevant input parameters and the (meta)modelling of inputs vs. outputs to understand their dependencies and interactions. In Fig. 1 a metamodel for the Mean Efficiency of a binary grating to couple a set of plane waves into a waveguide is shown as an example. It can be seen, that the coupling efficiency can be maximized by the Modulation Depth and Relative Slit Width at the same time. Dynardo's Metamodel of Optimal Prognosis has the ability to predict data points that haven't been used for modelling $[1,2]$.

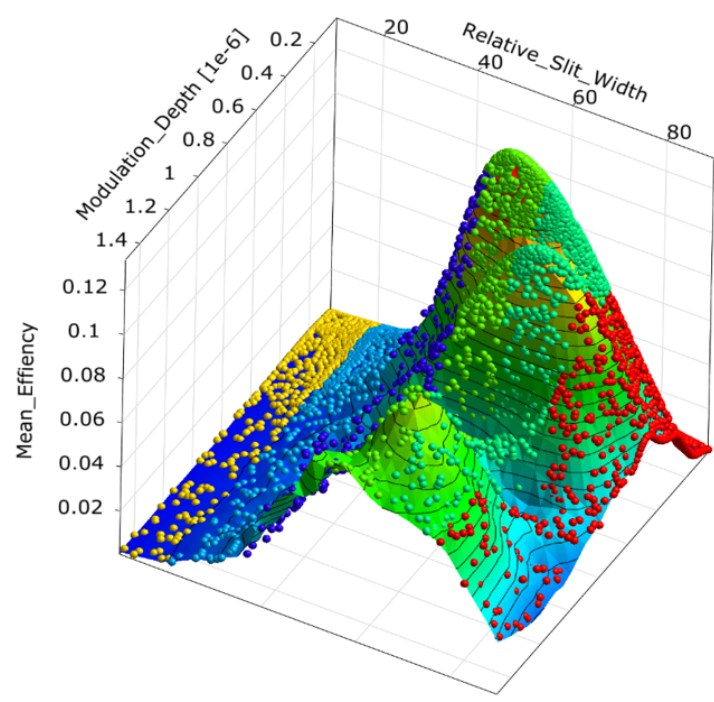

Fig. 1. Metamodel of Optimal Prognosis that shows the influence of the two input parameters Modulation Depth and Relative Slit Width on the Mean coupling Efficiency of the binary grating.

\subsection{Optimization}

The intelligent definition of objective functions for an efficient subsequent optimization is of high importance for multi-objective optimization tasks. Questions that can be answered using the results of the sensitivity analysis are:

- How to weight the objectives that are not in conflict to define them in one objective function? This can be defined based on the variation of the merits as result of the sensitivity analysis.

\footnotetext{
* Corresponding author: stephanie.kunath@dynardo.de
} 
- Are the objectives (merits) in conflict? In this case, they should be defined in separate objective functions.

To find the best trade-off between two or more merit functions, a Pareto optimization is the best choice. As a result, not only one design, but a front of best designs is obtained and the most appropriate design(s) can be selected by the decision maker. These best designs are usually plotted as Pareto front as shown in Fig. 2. Additionally, further understanding of the optical design can be obtained by clustering interesting parameters and colouring each of these resulting clusters. In Fig. 2 a cluster analysis of the Fill Factor of a slanted grating was considered to understand its influence on the performance (Uniformity Error as well as Mean Efficiency to couple a set of plane waves into a waveguide). It is obvious, that high fill factors lead to lower efficiencies. Furthermore, the trade-off between Uniformity Error and Mean Efficiency gets visible: the higher the efficiency the higher the uniformity error.

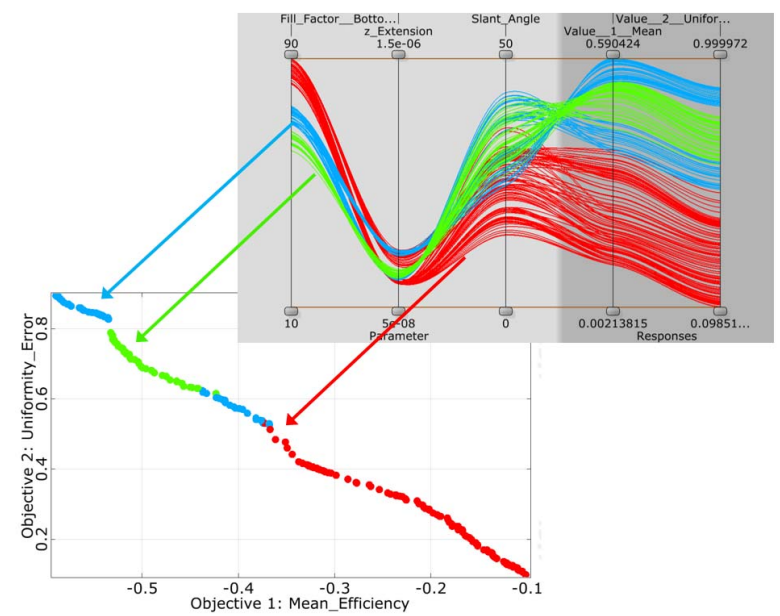

Fig. 2. Anthill plot with Pareto front designs (lower left plot) representing the trade-off between Uniformity Error and Mean Efficiency of a slanted grating. The colours correspond to the colour of the cluster analysis illustrated in the parallel coordinates plot (upper right plot). Here, the Fill Factor was separated into 3 clusters.

\subsection{Robustness analysis}

Besides the optimization of an optical design, its robustness is of high importance. That means, the optical performance should be rather independent on input parameter scattering due to tolerances, material parameter variation, heating etc. Therefore, the best trade-off between optimization and robustness targets needs to be found to secure the manufacturability of the optical design. Several strategies for Robust Design Optimization are available:

1. Iterative approach: first optimization, and then robustness analysis to

o reduce critical input scattering and/ or

o perform a subsequent optimization with changed constraints to find another, more robust optimum
2. Coupled approach: the optimizer contains optimization and robustness criteria as

o constraint or

o second objective function (Pareto optimization).

The results of such a coupled and fully automated Robust Design Optimization approach using a second objective function is demonstrated in Fig. 3. Here, a Cooke triplet was used as example. With this approach, the optical designer can make the final decision about the best optical design with other decision makers together by analysing the trade-off between optimization and robustness criteria. In this example, it gets obvious that the higher the performance in terms of optimization criteria, the worse the robustness of the design (higher values indicate higher variation of the performance).

With this approach, many iterative design steps can be saved. As a consequence, the multidisciplinary work between engineers of different physical domains, managers and the customer will be more efficient and transparent.

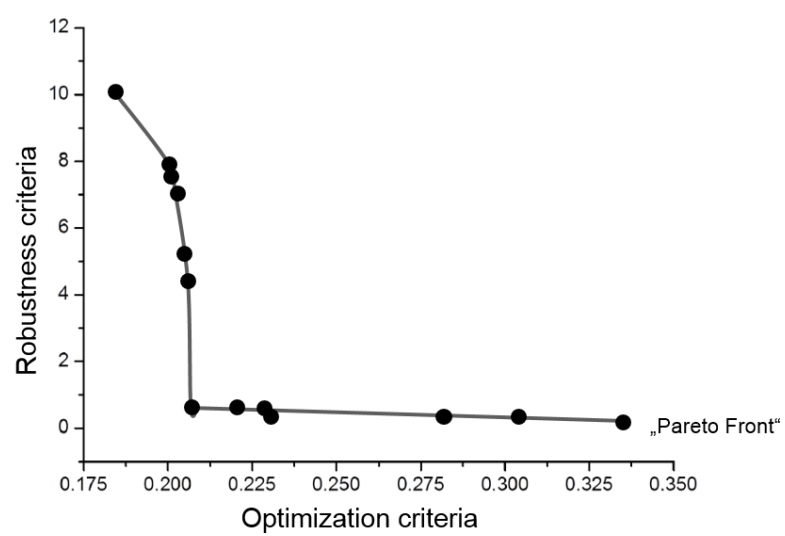

Fig. 3. Pareto Front as a result of a coupled Robust Design Optimization of a Cooke triplet. Two objective functions were used: one for the robustness criteria (here: weighted standard deviations of the merits) and a second one for the optimization criteria (here: weighted merits). Each design point on the front is a best design as a compromise between the two objectives.

\section{References}

1. Most, T. and J. Will (2011). Sensitivity analysis using the Metamodel of Optimal Prognosis, Proceedings Weimarer Optimization and Stochastic Days 8.0, Weimar, Germany

2. Most, T. and J. Will (2012). Robust Design Optimization in industrial virtual product development, Proceedings 5th International Conference on Reliable Engineering Computing, Brno, 2012 